\title{
Long-Term Risk of Progression to Sustained Hypertension in White-Coat Hypertension with Normal Night-Time Blood Pressure Values
}

\author{
João Faria $\mathbb{D}^{1},{ }^{1}$ José Mesquita Bastos $\mathbb{D},{ }^{2}$ Susana Bertoquini $\mathbb{D},{ }^{3}$ José Silva $\mathbb{D},{ }^{3}$ and \\ Jorge Polónia ${ }^{1}{ }^{1}$ \\ ${ }^{1}$ Department Medicine and Cintesis, Faculty of Medicine, University of Porto, Porto, Portugal \\ ${ }^{2}$ Health School of Aveiro University, Aveiro, Portugal \\ ${ }^{3}$ Hypertension Unit, Hospital Pedro Hispano, ULS, Matosinhos, Portugal
}

Correspondence should be addressed to Jorge Polónia; jjpolonia@gmail.com

Received 21 September 2020; Revised 11 November 2020; Accepted 10 December 2020; Published 22 December 2020

Academic Editor: Kai Hu

Copyright (c) 2020 João Faria et al. This is an open access article distributed under the Creative Commons Attribution License, which permits unrestricted use, distribution, and reproduction in any medium, provided the original work is properly cited.

\begin{abstract}
Background. The long-term prognosis and transition towards sustained ambulatory hypertension (SHT) of white-coat hypertension (WCHT) remain uncertain particularly in those with both normal nighttime and daytime blood pressure (BP) values. Different classification criteria and the use of antihypertensive drugs may contribute to conflicting results. Patients and Methods. We prospectively evaluated for a 7.1 year transition to SHT in 899 nondiabetic subjects free from cardiovascular (CV) events: normotensive (NT) ( $n=344 ; 52,9 \%$ female; ageing $48 \pm 14$ years); untreated WCHT (UnWCHT $n=399 ; 50,1 \%$ female; ageing $51 \pm 14$ years); and treated WCHT with antihypertensive drugs after baseline (TxWCHT $n=156 ; 54,4 \%$ female; ageing $51 \pm 15$ years). All underwent $24 \mathrm{~h}$ ambulatory BP monitoring (24h-ABPM) at baseline, at 30 to 60 months, and at 70 to 120 months thereafter. WCHT was at baseline (with no treatment) as office $\mathrm{BP} \geq 140 /$ or $90 \mathrm{~mm} \cdot \mathrm{Hg}$, daytime $\mathrm{BP}<135 / 85 \mathrm{~mm} \cdot \mathrm{Hg}$, and nighttime $\mathrm{BP}<120 / 70 \mathrm{~mm} \cdot \mathrm{Hg}$. Development of SHT was considered if daytime BP $\geq 135 /$ or $85 \mathrm{~mm} \mathrm{Hg}$ and/or nighttime $\mathrm{BP} \geq 120$ /or $70 \mathrm{~mm} \cdot \mathrm{Hg}$. Results. Baseline metabolic parameters did not differ among groups. At $30-60$ months and at the end of follow-up, development of SHT occurred, respectively, in NT $(3.8 \%(n=13)$ and $9.6 \%(n=33))$ and in UnWCHT $(10.1 \%(n=40)$ and $16.5 \%(n=66))(p<0.009)$. The mean annual increase of average $24 \mathrm{~h}$-systolic BP was $0.48+0.93$ in NT and $0.73+1.06$ in UnWCHT, whereas annual SBP in office increased in NT by $1.2+0.95$ but decreased in UnWCHT by $1.36+1.35 \mathrm{~mm} \mathrm{Hg}$ $(p<0.01)$. Conclusion. Untreated WCHT patients exhibit a faster and a higher risk of developing SHT compared to NT with TxWCHT assuming an intermediate position between them.
\end{abstract}

\section{Introduction}

White-coat hypertension (WCHT) refers to the untreated condition in which high blood pressure (BP) values in the office coexist with normal BP values when measured by $24 \mathrm{~h}$ ambulatory blood pressure monitoring (24-ABPM), home blood pressure monitoring (HBPM), or both [1]. From its recognition in 1987, until now, the clinical prognosis of WCHT remains uncertain. Early longitudinal studies suggested that WCHT would be an innocent entity proposing that WCHT carries a similar risk to normotension and significantly lower than sustained hypertension (SHT) [2-6] even after adjusting for age and cardiovascular (CV) risk factors [4, 7-11]. In contrast, other studies and meta-analyses suggest that WCHT carries a higher risk of CV morbidity and mortality and place it as an intermediate risk entity between NT and SHT [8, 10, 12-19]. However, overall these studies had a short duration and disparate inclusion criteria, particularly with regard to the definition of WCHT itself. In addition, in some studies, WCHT has been associated with a greater risk of transition for SHT, as well as overall increased risk of CV events [20-22]. Such contradictory results may be explained by the heterogeneity of the studied populations, definition of WCHT and inclusion of 
treated patients. In several studies, classification of WCHT has been based only on daytime BP values but not on nighttime $\mathrm{BP}$ (NBP) which is recognized to be a stronger predictor of cardiovascular risk [3, 23-25]. Moreover, whether or not patients with WCHT should receive antihypertensive drugs is also unresolved [1]. In the present study, our goal was to determine the long-term risk of individuals with WCHT with both normal daytime and NBP for progressing to SHT and the impact on it by the antihypertensive medication.

\section{Methods}

2.1. Patients and Protocols. We carried out a longitudinal observational study in a group of 989 outpatients selected at a hospital consultation from 3 hospitals in northern Portugal between 1991 and 2008. All subjects with BP>140/or $90 \mathrm{~mm} \cdot \mathrm{Hg}$ in office were referred by GPs to the Hospital Blood Pressure Units to perform $24 \mathrm{~h}$ ambulatory BP monitoring in order to confirm the diagnosis of sustained hypertension or of WCHT. The population of the present study was recruited from a cohort of 812 normotensives and 617 subjects with WCHT who were followed up for 7.9 years as previously described [8]. The patients were referred to the hospital consultation from the primary health care to perform a $24 \mathrm{~h}$ ambulatory blood pressure monitoring (24hABPM). A pool of normotensive subjects was also integrated into the study as previously described [8]. The protocol was carried out according to the WMA Declaration of Helsinki Ethical Principles for Medical Research Involving Human Subjects, and all data collection was approved by the local Hospital Ethical Committee. As in our previous study [8] to be included in the present one, patients had to be aged between 18 and 70 years and they should (i) have no history or clinical evidence of congestive heart failure, cerebrovascular disease, myocardial infarction, coronary bypass or angioplasty, cardiac valve disease, renal insufficiency, peripheral artery disease, atrial fibrillation or other major arrhythmias, or severe hepatic disease, (ii) have no clinical or analytical evidence of diabetes mellitus and of estimated glomerular filtration rate below $60 \mathrm{ml} / \mathrm{min} / 1.73 \mathrm{~m}^{2}$ (MDRD), and (iii) have neither suspicion of secondary hypertension nor clinical suspicion of sleep apnoea. WCHT was defined at baseline as follows: to be untreated at that time; with office $\mathrm{BP}$ of at least 140 or $90 \mathrm{~mm} \cdot \mathrm{Hg}$ in three previous consecutive recordings and also in the first measurement of the $24 \mathrm{~h}$ ABPM and with mean $24 \mathrm{~h}-\mathrm{ABPM}$ less than $130 / 80 \mathrm{~mm} \cdot \mathrm{Hg}$, daytime BP less than $135 / 85 \mathrm{~mm} \cdot \mathrm{Hg}$, and NBP less than 120 / $70 \mathrm{~mm} \cdot \mathrm{Hg}$. Thus subjects included in the present analysis who were diagnosed as WCHT were all absolutely untreated at baseline. After this initial selection, some WCHT were subsequently subjected to antihypertensive treatment (now designed as WCHT treated-TxWCHT) at the initiative of the respective GPs, while others remained untreated during the follow-up (now designed as WCHT untreated-UnWCHT). In the present study, we only included subjects from the previous cohort [8] that could be also revaluated with $24 \mathrm{~h}$ ABPM between 30 and 60 months and between 70 and 120 months after the first $24 \mathrm{~h}-\mathrm{ABPM}$ at baseline. Subjects with
WCHT who received prescriptions with any antihypertensive medications in any phase of the follow-up after the baseline (treated WCHT-TxWCHT) were evaluated separately from subjects with WCHT who were free of medication during the follow-up (untreated WCHT-UnWCHT). The decision to treat came from the patient's medical assistants, and the collection of information regarding previous antihypertensive medication (if any) was carried out at the moments of the two $24 \mathrm{~h}$ ambulatory BP evaluations during the follow-up.

2.2. Office and Ambulatory Blood Pressure Monitoring. Office BP was evaluated at admission in three different appointments. Brachial BP and heart rate were evaluated in the nondominant arm using an automated digital oscillometric sphygmomanometer (Omron, Model M6; Omron Corporation, Kyoto, Japan). Three readings, 2 min apart, were taken (on the same day) before the installation of the $24 \mathrm{~h}-\mathrm{ABPM}$, and the mean of the last two was considered the brachial BP. Twenty-four-hour ABPM was performed during a working day at the entry of the study with SpaceLabs 90207 (SpaceLabs Inc., Redmond, Washington, USA) as described earlier [8, 9, 26]. The monitor was mounted on the nondominant arm between 08:00 and 09: $00 \mathrm{~h}$ and was removed $24 \mathrm{~h}$ later. The patients were instructed to perform their usual daily activities and were asked to go to bed precisely at $23: 00 \mathrm{~h}$, to remain in bed until $07: 00 \mathrm{~h}$, and advised the use of an alarm clock to help wake up. This was confirmed on the basis of the information obtained from the patient's diary. BP was recorded every 20 min during the day (between 07:00 and 23:00 h) and every $30 \mathrm{~min}$ at night (between $23: 30$ and $06: 30 \mathrm{~h}$ ). The nocturnal SBP fall (\%) was calculated as $100 \mathrm{x}(1-$ sleep SBP/ awake SBP ratio), and the nocturnal DBP fall (\%) was calculated as $100 \mathrm{x}(1-$ sleep $\mathrm{DBP} /$ awake DBP ratio). These periods were considered to be representative of the awake and nighttime resting BPs, respectively.

2.3. Follow-Up and End Point Evaluation. The patient's medical records were reviewed for the use of antihypertensive drug therapy and further transition to SHT at the time of the two ABPM recordings during the follow-up which occurred from January 1991 to December 2013. All subjects had beginning normal values of both daytime and nighttime BP. The endpoint was the transition to SHT, defined during the follow-up by the presence at any of the two moments of evaluation of a 24-ABPM, a daytime $\mathrm{BP} \geq 135 / 85 \mathrm{~mm} \cdot \mathrm{Hg}$ and/or $\mathrm{NBP} \geq 120 / 70 \mathrm{~mm} \cdot \mathrm{Hg}$ either in the populations of normotensives, WCTH untreated, and WCHT treated. As in the previous study [8] the presence/ absence of CV events was confirmed by the examination of the patient's medical records until the end of the follow-up period. Cardiovascular events were diagnosed either by the physician who cared for the patients at the time of the events or if death had occurred; information on its cause was obtained either from the patient's physician or otherwise by examination of the official death certificate. In all cases, the diagnosis of $\mathrm{CV}$ events was confirmed objectively by an 
external expert who examined the patient's records and diagnostic procedures. Both fatal and nonfatal cardiovascular events considered consisted of congestive heart failure, cerebrovascular disease, myocardial infarction, angina pectoris, coronary bypass, or angioplasty. Coronary events included sudden death and fatal and nonfatal myocardial infarction or angina pectoris confirmed in the hospital and coronary bypass or angioplasty. Transient ischaemic attack was not considered an event.

2.4. Statistical Analysis. Statistical analysis was carried out using the SPSS software (version 13.0; SPSS Inc., Chicago, Illinois, USA). Values of continuous variables are presented as the mean $\pm S D$ categorized data as percentages, and differences between the groups were evaluated by one-way analysis of variance. Continuous variables were compared using one-way ANOVA with Tukey Kramer post hoc test. Nonnormal distribution data were assessed by Kruskal-Wallis nonparametric test. Chi-square test was used for group comparisons for categorized data. Statistical significance was considered for a $p$ value less than 0.05 .

\section{Results}

3.1. Study Population. We evaluated at baseline and at the two moments of the follow-up a total of 899 subjects who were 344 normotensives and 555 subjects with WCHT including 399 untreated and 156 treated with antihypertensive drugs. They were selected from a population studied in a previous cohort [8] but among these, we analysed only those who had available and absolutely reliable data of an evaluation with $24 \mathrm{~h}-\mathrm{ABPM}$ between 30 and 60 months and between 70 and 120 months after the first $24 \mathrm{~h}-\mathrm{ABPM}$ at baseline. Thus, from the previous cohort [8] and under those criteria, we analysed 344 out of 812 normotensives and 555 out of 617 subjects with WCHT, i.e., including only those who had available two additional ABPM recordings during the follow-up. Out of the 156 treated subjects with WCHT, 48 subjects received treatment before the $30^{\text {th }}$ month of follow-up, and the remaining 108 subjects thereafter until the end of the follow-up (see Section 3.2).

3.2. Flow Chart. The median follow-up period was $7.1+1.9$ years (0.9-10.1). Treatment consisted on: angiotensin receptor blockers (ARBs) single dose $(n=54)$, ARB combined with hydrochlorothiazide, $(n=6)$, angiotensin-converting enzyme inhibitors (ACEin) single dose $(n=22)$, ACEin combined with diuretics $(n=27)$, calcium antagonists single dose $(n=20)$; beta-blockers single dose, $(n=19)$ and Indapamide single dose $(n=8)$. Table 1 represents the clinical data of the three populations. As shown, subjects with WCHT were slightly older than normotensives and treated WCHT had a higher serum uric acid than the other groups. For the other clinical variables, groups did not differ significantly. Table 2 shows for the three groups the blood pressure values in office and with $24 \mathrm{~h} \mathrm{ABPM}$ both at baseline and at the end of the follow-up as well as the differences of BP calculated between these moments. At baseline, both groups of WCHT had similar BP values, whereas normotensives had lower office SBP and DBP, $24 \mathrm{~h}$ SBP and DBP, daytime SBP, NBP, and lower nighttime fall of SBP than both the WCHT groups. At the end of follow-up, both normotensives and treated WCHT had lower SBP and DBP at the office, $24 \mathrm{~h}$, daytime and nighttime than untreated WCHT. At the end of the follow-up, ambulatory BP values of normotensives and treated WCHT were not different but office BP was lower in normotensives. At baseline, the standard deviation (SD) of both $24 \mathrm{~h} \mathrm{SBP}$ and DBP, as an estimation of BP variability, was significantly lower in the normotensive group (SD of 24 SBP $11.9+2.8$ and SD $24 \mathrm{~h}$ DBP $10.1+2.2$ ) as compared with the WCHT group (SD of $24 \mathrm{~h} \mathrm{SBP} 14.4+3.2$ and SD $24 \mathrm{~h} \mathrm{DBP} 10.9+3.6)(p<0.01)$ for both, respectively.

Concerning the differences (delta) of BP values between baseline and the end of the follow-up, Table 2 shows that office BP increased in normotensives but decreased in subjects with WCHT particularly in the subgroup of those who were treated. From baseline to the end of follow-up, the net increases of $24 \mathrm{~h}$; daytime and nighttime SBP values were significantly lower in treated WCHT than in untreated WCHT. Figure 1 shows the percentage of subjects who progressed to sustained hypertension.

Both at the end and between 30-60 months of the followup, the percentage of normotensives that progressed to SHT was significantly lower than that of subjects with untreated WCHT. Regarding the totality of individuals who at the end of the follow-up progressed to sustained hypertension, in the first assessment at 30-60 months of the follow-up such a progression occurred only in $40 \%$ of normotensives, whereas it already occurred in $61 \%$ of the untreated WCHT. In other words, in the first assessment at 30-60 months of the followup, the percentage of subjects who showed a transition to SHT of untreated subjects with WCHT was 2.7 times higher than normotensives. At the end of the follow-up, these values were 1.7 times vs. that of normotensives. Among subjects who evolved to SHT during the follow-up, this was observed either at the expense of only daytime BP, or at the expense of only nighttime BP, or at the expense of both daytime and nighttime BP. That was observed, respectively, in $11(36 \%), 9(24 \%)$, and $13(40 \%)$ out of 33 subjects with NT and, respectively. in 28 (38\%), 18 (29\%), and 20 (33\%) out of 66 subjects with UnWCHT.

Table 3 shows the annual evolution of BP during the follow-up in the three groups. In normotensives, the annual change of office BP tended to increase. In contrast, it decreased in both treated and untreated WCH but significantly more in treated WCHT than in untreated WCHT. Untreated WCHT showed a higher annual increase of $24 \mathrm{~h}$, daytime, and NBP than untreated WCHT. Normotensives showed a lower annual increase of $24 \mathrm{~h} \mathrm{BP}$ than untreated $\mathrm{WCH}$ but a higher annual increase of $24 \mathrm{~h}$ BP than treated WCHT. During the follow-up, there were in the normotensives 16 $(4.7 \%)$ cardiovascular events (10 strokes and 6 coronary episodes), in the untreated WCHT 28 (7\%) events (18 strokes and 10 coronary episodes), and in treated WCHT 13 ( $8 \%)$ events (5 strokes and 8 coronary episodes), $p=0.188$, among all. All the events were nonfatal. 
TABle 1: Anthropometric biochemical data at baseline.

\begin{tabular}{|c|c|c|c|c|}
\hline & $\mathrm{NT}(n=434)$ & WCHT untreated $(n=399)$ & WCHT treated $(n=156)$ & $p=$ \\
\hline Age (years) & $49 \pm 14$ & $51 \pm 14^{\#}$ & $51 \pm 15^{\#}$ & 0.011 \\
\hline Female (\%) & 52.9 & 51.1 & 52.4 & 0.665 \\
\hline BMI $\left(\mathrm{Kg} / \mathrm{m}^{2}\right)$ & $27 \pm 3$ & $26 \pm 4$ & $26 \pm 4$ & 0.229 \\
\hline Smoking (\%) & 18 & 16 & 14 & 0.564 \\
\hline Glucose (mg/dl) & $91 \pm 022$ & $97 \pm 28$ & $98 \pm 25$ & 0.171 \\
\hline Creatinine (mg/dl) & $1.06 \pm 0.27$ & $0.99 \pm 0.31$ & $1.03 \pm 0.40$ & 0.783 \\
\hline Sodium $(\mathrm{mEq} / \mathrm{L})$ & $139 \pm 2$ & $140 \pm 3$ & $140 \pm 2$ & 0.123 \\
\hline Potassium (mEq/L) & $4.4 \pm 0.8$ & $4.3 \pm 0.5$ & $4.5 \pm 0.10$ & 0.160 \\
\hline Uric acid $(\mathrm{mg} / \mathrm{ml})$ & $4.9 \pm 1.4$ & $5.2 \pm 1.6$ & $5.4 \pm 1.7^{*}$ & 0.021 \\
\hline Total cholesterol (mg/dl) & $197 \pm 35$ & $199 \pm 38$ & $204 \pm 39$ & 0.259 \\
\hline HDL-C $(\mathrm{mg} / \mathrm{dl})$ & $56 \pm 14$ & $56 \pm 15$ & $56 \pm 16$ & 0.864 \\
\hline LDL-C (mg/dl) & $118 \pm 33$ & $119 \pm 34$ & $121 \pm 34$ & 0.320 \\
\hline Triglycerides (mg/dl) & $117 \pm 44$ & $122 \pm 49$ & $121 \pm 45$ & 0.793 \\
\hline Albuminuria (mg/24h) & $31 \pm 17$ & $32 \pm 21$ & $29 \pm 20$ & 0.546 \\
\hline
\end{tabular}

NT, normotensives; WCHT, white-coat hypertensives; BMI, body mass index. Post hoc analysis: ${ }^{*} p<0.05$ vs. NT and WCHT treated; ${ }^{\#} p<0.05$ vs. NT.

In the overall population and in all the subpopulations (NT, WCHT treated, and nontreated), there were no differences between individuals with or without transition to SHT regarding anthropometric (age, BMI, and gender) and biochemical parameters. However, in all populations, those with transition to SHT showed systematically significant higher values of systolic BP in office, 24 hours, daytime, and nighttime in comparison to those without transition to SHT. For example, in overall population, systolic BP values either in office, 24 hours, daytime, and nighttime were higher in subjects with transition to SHT $(n=122)$ vs. without transition to SHT $(n=767)$ i.e., respectively, $147+15$ vs. $142+15,123+5$ vs. $121+6,128+7$ vs. $125+7$, and $113+7$ vs. $111+6 \mathrm{~mm} \mathrm{Hg}$ (all $p<0.01)$.

\section{Discussion}

The aim of the present study was to evaluate the cumulative incidence of SHT in subjects with treated and untreated WCHT compared to normotensive subjects over 7.1 years of follow-up. Our main findings are that untreated subjects with WCHT defined by normalcy of both daytime and nighttime $\mathrm{BP}$ values show an increased risk of transition to SHT and an earlier progression to SHT in comparison to normotensive subjects. However, we cannot exclude that the higher age and baseline BP values of WCHT vs. NT may have influenced the greater propensity for the development of hypertension of WCHTs. At baseline, the SD of both $24 \mathrm{~h}$ SBP and $24 \mathrm{~h}$ DBP was significantly lower in the normotensive group as compared with the WCHT group which may also have contributed to the lower rate of progression of normotensives to sustained hypertension in comparison with WCHT. Treated WCHT appears to assume an intermediate position between untreated WCHT and NT thereby showing an annual evolution of BP values that were lower than both untreated WCHT and normotensives. In all populations (all together and in subgroups) those with a transition to SHT showed at baseline systematically significant higher values of systolic BP in office, 24 hours, daytime, and nighttime in comparison to those without transition to SHT. Thus we cannot exclude that higher baseline BP may represent an important determinant of the evolution to SHT. Another striking point of the study besides the classification of WCHT was the evaluation of 24-ABPM during two distinct moments of the follow-up that allowed us to establish a temporal evolution of the transition to SHT. We found that, at 30-60 months of the follow-up, the transition to SHT of untreated WCHT occurred earlier, being 2.7 times more frequent than normotensives. At the end of the followup, these values were about 1.7 times that of normotensives. Again, treated WCHT appears to assume an intermediate position between untreated WCHT and NT. The CV prognosis of WCHT and the risk of transition to SHT are still controversial. The alleged benignity of WCHT has been challenged and its long-term CV risk has been considered to be intermediate between NT and SHT [12, 14, 18, 27]. We hypothesised that an incomplete classification of individuals with WCHT may have contributed towards the conflicting data among the different studies. In fact, most of the related studies have used office BP $(\geq 140 / 90 \mathrm{~mm} \cdot \mathrm{Hg})$ and out-ofoffice BP $(\leq 130-135 / 80-85 \mathrm{~mm} \cdot \mathrm{Hg})$ thereby avoiding considering NBP values [6, 27-30]. In other words, nocturnal $\mathrm{BP}$, which has been related strongly to the $\mathrm{CV}$ prognosis $[8,23,24,31,32]$ was not considered in the WCHT definition in most of the available evidence, and this may have conditioned the results. We have recently found [8] in a followup of 7.4 years that WCHT patients who were classified on the basis of normal $24 \mathrm{~h}$, daytime, and NBP showed a CV prognosis not different from NT but better than true hypertensives. This suggests that normal levels of NBP should be included in its classification to avoid misdiagnosis of WCHT, as suggested by some authors [33]. In general, a better prognosis of WCHT should be expected when normal NBP values are included in the definition of WCHT since NBP is a stronger predictor of risk than daytime BP [25]. In fact, a lower prevalence rate of WCHT $[6,34-36]$ and a lower value of left ventricular mass index [29] were found when normal values of daytime and nighttime $\mathrm{BP}$ values were considered together in the classification of WCHT compared with the condition of including daytime BP only. Moreover, the prevalence of WCHT was reduced when NBP was used for its categorization [36]. In the present study, as in our 
TABLE 2: Blood pressure data.

\begin{tabular}{|c|c|c|c|c|}
\hline & NT $(n=434)$ & WCHT untreated $(n=399)$ & WCHT treated $(n=156)$ & $p \leq$ \\
\hline \multicolumn{5}{|l|}{ In office-blood pressure and heart rate } \\
\hline SBPc baseline (mm Hg) & $128 \pm 8$ & $151 \pm 11^{*}$ & $150 \pm 10^{*}$ & 0.001 \\
\hline DBPc baseline (mm Hg) & $84 \pm 9$ & $91 \pm 10^{*}$ & $90 \pm 10^{*}$ & 0.001 \\
\hline HRc baseline (b/min) & $78 \pm 10$ & $81 \pm 9$ & $80 \pm 10$ & 0.710 \\
\hline SBPc final (mm Hg) & $131 \pm 8.9$ & $146 \pm 11^{*}$ & $143 \pm 10^{*}$ & 0.001 \\
\hline DBPc final (mm Hg) & $86 \pm 9$ & $88 \pm 10^{*}$ & $86 \pm 10^{*}$ & 0.001 \\
\hline HRc final $(\mathrm{b} / \mathrm{min})$ & $79 \pm 11$ & $80 \pm 9$ & $79 \pm 11$ & 0.261 \\
\hline Delta SBPc (mm hg) & $3.0 \pm 2.1$ & $-3.9 \pm 3.0^{*}$ & $-6.1 \pm 5.9^{*}$ & 0.001 \\
\hline Delta DBPc (mm hg) & $2.8 \pm 3.7$ & $-2.6 \pm 2.7^{*}$ & $-4.2 \pm 2.1^{*}$ & 0.001 \\
\hline \multicolumn{5}{|l|}{24 h-blood pressure and heart rate } \\
\hline SBP $24 \mathrm{~h}$ baseline $(\mathrm{mm} \mathrm{Hg})$ & $120 \pm 7$ & $123 \pm 5^{*}$ & $122 \pm 5^{*}$ & 0.001 \\
\hline DBP $24 \mathrm{~h}$ baseline $(\mathrm{mm} \mathrm{Hg})$ & $73 \pm 7$ & $75 \pm 7^{*}$ & $74 \pm 7$ & 0.001 \\
\hline HR $24 \mathrm{~h}$ baseline $(\mathrm{b} / \mathrm{min})$ & $74 \pm 9$ & $73 \pm 10$ & $72 \pm 10$ & 0.159 \\
\hline SBP 24 h final (mm Hg) & $122 \pm 7$ & $125 \pm 6^{*}$ & $123 \pm 6^{\S}$ & 0.001 \\
\hline DBP $24 \mathrm{~h}$ final $(\mathrm{mm} \mathrm{Hg})$ & $74 \pm 6$ & $78 \pm 7^{*}$ & $73 \pm 6^{\S}$ & 0.001 \\
\hline HR $24 \mathrm{~h}$ final (b/min) & $73 \pm 8$ & $72 \pm 9$ & $72 \pm 9$ & 0.311 \\
\hline Delta SBP24 h (mm Hg) & $2.4 \pm 3.1$ & $2.9 \pm 4.1^{* \#}$ & $0.7 \pm 1.9^{* \S}$ & 0.001 \\
\hline Delta DBP24h (mm Hg) & $1.1 \pm 2.4$ & $1.2 \pm 5.4^{\#}$ & $0.23 \pm 1.4^{* \S}$ & 0.001 \\
\hline \multicolumn{5}{|l|}{ Daytime-blood pressure and heart rate } \\
\hline SBP daytime baseline $(\mathrm{mm} \mathrm{Hg})$ & $124 \pm 8$ & $126 \pm 6^{*}$ & $126 \pm 5^{*}$ & 0.01 \\
\hline DBP daytime baseline (mm Hg) & $77 \pm 7$ & $79 \pm 7^{*}$ & $78 \pm 7$ & 0.01 \\
\hline HR daytime baseline $(\mathrm{b} / \mathrm{min})$ & $77 \pm 10$ & $76 \pm 11$ & $75 \pm 10$ & 0.170 \\
\hline SBP daytime final $(\mathrm{mm} \mathrm{Hg})$ & $126 \pm 8$ & $129 \pm 7^{* \#}$ & $127 \pm 6$ & 0.001 \\
\hline DBP daytime final (mm Hg) & $78 \pm 7$ & $80 \pm 7^{* \#}$ & $77 \pm 7$ & 0.01 \\
\hline HR daytime final (b/min) & $76 \pm 9$ & $77 \pm 10$ & $74 \pm 10$ & 0.231 \\
\hline Delta SBP daytime (mm Hg) & $2.2 \pm 3.1$ & $2.5 \pm 6.0^{*} \#$ & $0.7 \pm 2.8^{* \S}$ & 0.001 \\
\hline Delta DBP daytime (mm Hg) & $1.0 \pm 2.5$ & $1.5 \pm 6.1^{*} \#$ & $0.4 \pm 1.8^{* \S}$ & 0.001 \\
\hline \multicolumn{5}{|c|}{ Nighttime-blood pressure and heart rate } \\
\hline SBP nighttime baseline (mm Hg) & $110 \pm 8$ & $111 \pm 7$ & $112 \pm 8^{*}$ & 0.01 \\
\hline DBP nighttime baseline $(\mathrm{mm} \mathrm{Hg})$ & $65 \pm 7$ & $66 \pm 7^{*}$ & $66 \pm 7^{*}$ & 0.01 \\
\hline HR nighttime baseline $(\mathrm{b} / \mathrm{min})$ & $66 \pm 9$ & $65 \pm 9$ & $66 \pm 10$ & 0.644 \\
\hline SBP nighttime final (mm Hg) & $112 \pm 9$ & $114 \pm 8^{*}$ & $112 \pm 8$ & 0.013 \\
\hline DBP nighttime final (mm Hg) & $66 \pm 7$ & $67 \pm 8$ & $65 \pm 7$ & 0.128 \\
\hline HR nighttime final $(\mathrm{b} / \mathrm{min})$ & $67 \pm 9$ & $66 \pm 9$ & $67 \pm 10$ & 0.233 \\
\hline Delta SBP nighttime (mm Hg) & $1.7 \pm 3.0$ & $2.1 \pm 9.0^{* \#}$ & $0.5 \pm 3.0^{* \S}$ & 0.001 \\
\hline Delta DBP nighttime (mm Hg) & $0.6 \pm 4.5$ & $0.9 \pm 6.9^{* \#}$ & $0.7 \pm 2.8$ & 0.01 \\
\hline \multicolumn{5}{|l|}{ Nighttime BP fall (\%) } \\
\hline Nighttime fall SBP baseline & $10.7 \pm 5.9$ & $12.1 \pm 6.5^{*}$ & $12.7 \pm 6.8^{*}$ & 0.01 \\
\hline Nighttime fall DBP baseline & $15.3 \pm 7.4$ & $15.9 \pm 7.6$ & $16.3 \pm 7.6$ & 0.259 \\
\hline Nighttime fall SBP final & $10.3 \pm 4.9$ & $11.9 \pm 5.8^{*}$ & $12.0 \pm 5.9^{*}$ & 0.01 \\
\hline Nighttime fall DBP final & $15.1 \pm 6.5$ & $15.3 \pm 6.8$ & $15.4 \pm 6.8$ & 0.232 \\
\hline
\end{tabular}

NT, normotensives; WCHT, white-coat hypertensives; SBPc, office systolic BP; DBPc, office diastolic BP; HRc, office heart rate; Delta, difference between baseline and the end of the follow-up (final). Post hoc analysis: ${ }^{*} p<0.01$ vs. NT; ${ }^{\#} p<0.01$ different from WCHT treated; ${ }^{{ }^{* *}} p<0.05$ WCHT treated vs. WCTH untreated.

previous one [8], we have failed to demonstrate a clearly higher rate of events in the WCHT than in normotensives. However, because of the small number of events in differences between NT and WCHT populations a type 2 error cannot be ruled out. We found at the end of the follow-up a cumulative incidence of SHT in our WCHT populations between $10.5-16.5 \%$ which were significantly higher than in the normotensive population. In larger series, a $40 \%$ or higher propensity of WCHT to SHT was reported by others $[20,37,38]$. Some studies [39] found in WCHT of a greater prevalence of metabolic risk factors and a greater long-term risk of new-onset diabetes and a progression to sustained hypertension. In contrast, in our study at baseline anthropometric and biochemical characteristics and percentage of smokers of our WCHT population did not differ from that of normotensives which may also explain the relatively benign outcome and the lower percentage of transition to SHT of our WCHT population. Nevertheless, our data still support the prudent recommendations $[1,22,32,34]$ in relation to this entity of close surveillance with a frequent BP measurement outside the office. Since $24 \mathrm{~h}-\mathrm{ABPM}$ enlightens the nighttime $\mathrm{BP}$ values, it is the preferential tool for the definition of WCHT unless new devices to home $\mathrm{BP}$ able to register the NBP will become available. There is also some debate whether drug therapy may influence the long-term evolution of $\mathrm{BP}$ and the $\mathrm{CV}$ 


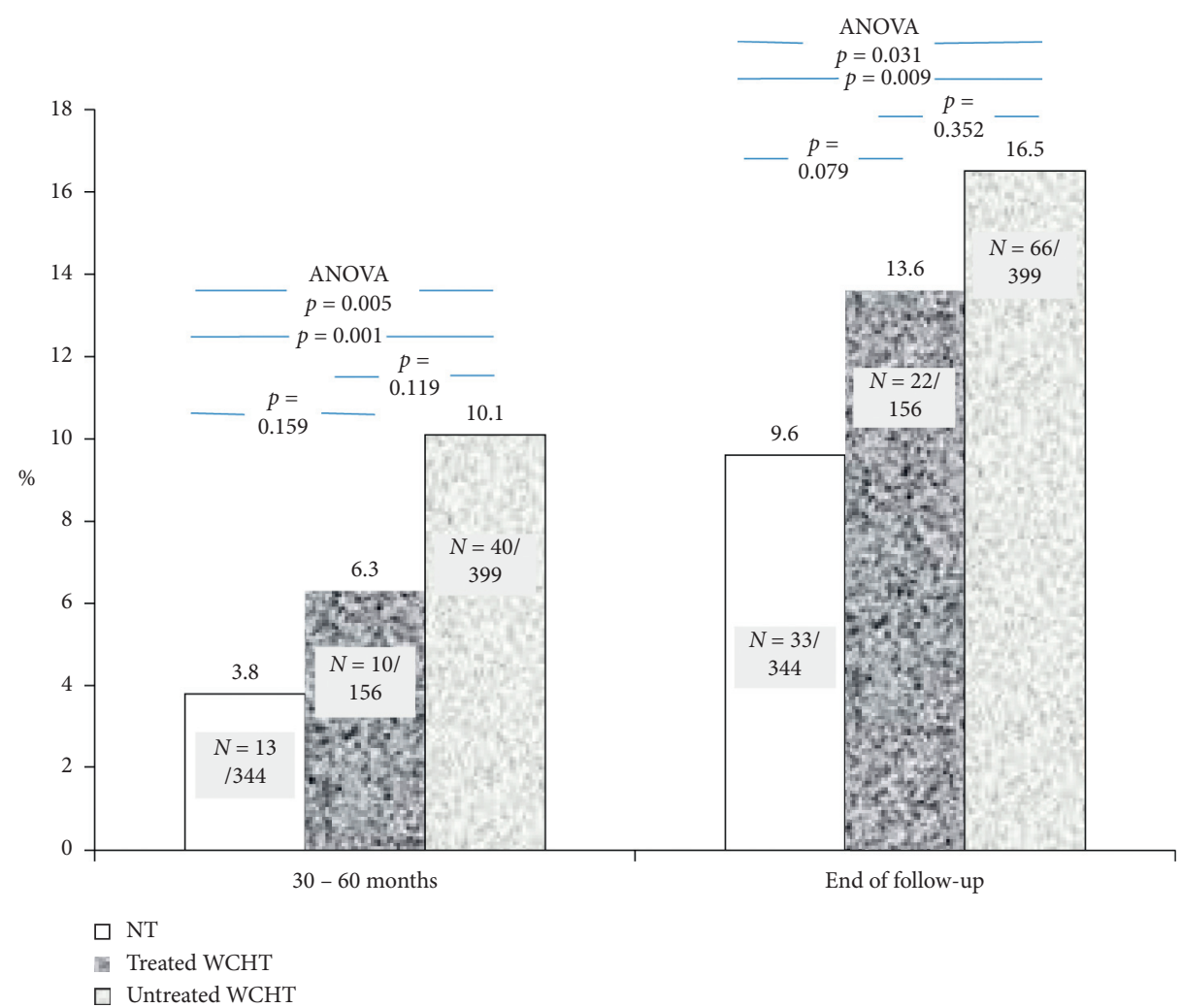

FIGURE 1: Cumulative incidence and rate (\%) of progression to sustained hypertension (SHT) of normotensive subjects (NT) and treated and untreated WCHT subjects. In untreated WCHT, SHT occurred more frequently and earlier than NT.

TABle 3: Annual evolution of BP (mm Hg/year) during the follow-up.

\begin{tabular}{|c|c|c|c|c|}
\hline & NT $(n=434)$ & WCHT untreated $(n=399)$ & WCHT treated $(n=156)$ & ANOVA $p<$ \\
\hline $\mathrm{SBPc}(\mathrm{mm} \mathrm{Hg})$ & $1.2 \pm 0.95$ & $-1.36 \pm 1.35^{* \#}$ & $-2.03 \pm 1.56^{*}$ & 0.01 \\
\hline $\mathrm{DBPc}(\mathrm{mm} \mathrm{Hg})$ & $1.01 \pm 0.77$ & $-0.98 \pm 0.97^{\#}$ & $-1.13 \pm 0.75$ & 0.01 \\
\hline SBP $24 \mathrm{~h}(\mathrm{~mm} \mathrm{Hg})$ & $0.53 \pm 0.93$ & $0.76 \pm 1.06^{* \#}$ & $0.35 \pm 1.01$ & 0.01 \\
\hline DBP 24 h (mm Hg) & $0.22 \pm 0.95$ & $0.39 \pm 1.11^{* \#}$ & $0.12 \pm 0.71$ & 0.01 \\
\hline SBP daytime $(\mathrm{mm} \mathrm{Hg})$ & $0.54 \pm 1.00$ & $0.58 \pm 1.48^{\#}$ & $0.37 \pm 1.34$ & 0.01 \\
\hline DBP daytime $(\mathrm{mm} \mathrm{Hg})$ & $0.28 \pm 1.05$ & $0.38 \pm 1.94^{\#}$ & $0.11 \pm 0.92$ & 0.01 \\
\hline SBP nighttime (mm Hg) & $0.54 \pm 0.84$ & $0.63 \pm 1.03^{\#}$ & $0.33 \pm 0.92$ & 0.01 \\
\hline DBP nighttime (mm Hg) & $0.21 \pm 0.89$ & $0.33 \pm 1.00^{\#}$ & $0.11 \pm 0.87$ & 0.01 \\
\hline
\end{tabular}

NT, normotensives; WCHT, white-coat hypertensives; SBPc, office systolic BP; DBPc, office diastolic BP; HRc, office heart rate; Delta, difference between baseline and the end of the follow-up (final). Post hoc analysis: ${ }^{*} p<0.01$ vs. NT ${ }^{\#} p<0.01$ different from WCHT treated.

outcomes of WCHT and whether or not patients with WCHT should receive antihypertensive therapy $[7,17,36,40,41]$. We were unable to contribute to clarify that dispute in view of the limited number of our TxWCHT subjects. To our knowledge, our study is one of the first studies that have prospectively studied WCHT classified on the basis of normal NBP and examined the long-term transition of to SHT using two moments of $24 \mathrm{~h}-\mathrm{ABPM}$ after the baseline. Our study must be interpreted within the context of its strengths and potential limitations. First, the participants were selected from a regional database so that the results may not be extrapolated at a national or an international level. Second, because we were unable to evaluate all biochemical and anthropometric data during and at the end of the following, we cannot exclude that throughout that period changes in habits and behaviours may have influenced both office and ambulatory BP.

\section{Conclusion}

In our WCHT population selected by normalcy of daytime and NBP values, we found a faster and a significantly higher risk of transition from WCHT to SHT compared to normotensive subjects. Treated WCHT appears to assume an intermediate position between untreated WCHT and NT. Although we still lack robust evidence to confirm the benefit 
of antihypertensive treatment in the outcome of WCHT, the transition to SHT may be attenuated or delayed when antihypertensive therapy is undertaken. Although no increase in cardiovascular events was so far detected, these results support that WCHT subjects should be closely monitored for control of associated risk factors and early detection of progression to SHT, desirably with 24-ABPM.

\section{Data Availability}

Data are available in the Blood Pressure Unit Hospital Pedro Hispano, Matosinhos Portugal.

\section{Conflicts of Interest}

The authors declare that they have no conflicts of interest.

\section{Acknowledgments}

This study was supported by National Funds through the FCT-Fundação para a Ciência e a Tecnologia, I.P., within CINTESIS, R\&D Unit (reference: UIDB/4255/2020).

\section{References}

[1] B. Williams, G. Mancia, W. Spiering et al., "ESC/ESH Guidelines for the management of arterial hypertension," European Heart Journal, vol. 39, no. 33, pp. 3021-3104, 2018.

[2] H. Celis, J. A. Staessen, L. Thijs et al., "Cardiovascular risk in white-coat and sustained hypertensive patients," Blood Pressure, vol. 11, no. 6, pp. 352-356, 2002.

[3] K. Kario, K. Shimada, J. E. Schwartz, T. Matsuo, S. Hoshide, and T. G. Pickering, "Silent and clinically overt stroke in older Japanese subjects with white-coat and sustained hypertension," Journal of the American College of Cardiology, vol. 38, no. 1, pp. 238-245, 2001.

[4] R. S. Khattar, R. Senior, and A. Lahiri, "Cardiovascular outcome in white-coat versus sustained mild hypertension," Circulation, vol. 98, no. 18, pp. 1892-1897, 1998.

[5] G. Mancia, G. Bertinieri, G. Grassi et al., "Effects of bloodpressure measurement by the doctor on patient's blood pressure and heart rate," The Lancet, vol. 322, no. 8352, pp. 695-698, 1983.

[6] S. Omboni, "A working definition of white-coat hypertension must include nocturnal blood pressure," The Journal of Clinical Hypertension, vol. 32, 2018.

[7] R. H. Fagard, J. A. Staessen, L. Thijs et al., "Response to antihypertensive therapy in older patients with sustained and nonsustained systolic hypertension. Systolic Hypertension in Europe (Syst-Eur) Trial Investigators," Circulation, vol. 102, no. 10, pp. 1139-1144, 2000.

[8] J. Faria, J. Mesquita-Bastos, S. Bertoquini, J. Silva, L. Barbosa, and J. Polónia, "Long-term cardiovascular risk of white-coat hypertension with normal night-time blood pressure values," Blood Pressure Monitoring, vol. 24, no. 2, pp. 59-66, 2019.

[9] A. Groppelli, S. Omboni, G. Parati, and G. Mancia, "Evaluation of noninvasive blood pressure monitoring devices Spacelabs 90202 and 90207 versus resting and ambulatory 24hour intra-arterial blood pressure," Hypertension, vol. 20, no. 2, pp. 227-232, 1992.

[10] P. Verdecchia, G. P. Reboldi, F. Angeli et al., "Short- and longterm incidence of stroke in white-coat hypertension," $\mathrm{Hy}$ pertension, vol. 45, no. 2, pp. 203-208, 2005.
[11] R. H. Fagard and V. A. Cornelissen, "Incidence of cardiovascular events in white-coat, masked and sustained hypertension versus true normotension: a meta-analysis," Journal of Hypertension, vol. 25, no. 11, pp. 2193-2198, 2007.

[12] A. Briasoulis, E. Androulakis, M. Palla, N. Papageorgiou, and D. Tousoulis, "White-coat hypertension and cardiovascular events," Journal of Hypertension, vol. 34, no. 4, pp. 593-599, 2016.

[13] J. B. Cohen, M. J. Lotito, U. K. Trivedi, M. G. Denker, D. L. Cohen, and R. R. Townsend, "Cardiovascular events and mortality in white coat hypertension," Annals of Internal Medicine, vol. 170, no. 12, pp. 853-862, 2019.

[14] P. H. Gustavsen, A. Høegholm, L. E. Bang, and K. S. Kristensen, "White coat hypertension is a cardiovascular risk factor: a 10-year follow-up study," Journal of Human Hypertension, vol. 17, no. 12, pp. 811-817, 2003.

[15] Y. Huang, W. Huang, and Y. Hu, "Is white-coat hypertension associated with risk of cardiovascular diseases?" Journal of the American College of Cardiology, vol. 69, no. 23, p. 2880, 2017.

[16] S. D. Pierdomenico and F. Cuccurullo, "Prognostic value of white-coat and masked hypertension diagnosed by ambulatory monitoring in initially untreated subjects: an updated meta analysis," American Journal of Hypertension, vol. 24, no. 1, pp. 52-58, 2011.

[17] G. S. Stergiou, K. Asayama, L. Thijs et al., "Prognosis of whitecoat and masked hypertension," Hypertension, vol. 63, no. 4, pp. 675-682, 2014.

[18] G. Tocci, V. Presta, I. Figliuzzi et al., "Prevalence and clinical outcomes of white-coat and masked hypertension: analysis of a large ambulatory blood pressure database," The Journal of Clinical Hypertension, vol. 20, no. 2, pp. 297-305, 2018.

[19] S. D. Pierdomenico, A. M. Pierdomenico, F. Coccina, and E. Porreca, "Prognosis of masked and white coat uncontrolled hypertension detected by ambulatory blood pressure monitoring in elderly treated hypertensive patients," American Journal of Hypertension, vol. 30, no. 11, pp. 1106-1111, 2017.

[20] I. Bidlingmeyer, M. Burnier, M. Bidlingmeyer, B. Waeber, and H. R. Brunner, "Isolated office hypertension: a prehypertensive state?" Journal of Hypertension, vol. 14, no. 3, pp. 327-332, 1996.

[21] C. Cuspidi, M. Rescaldani, M. Tadic, C. Sala, G. Grassi, and G. Mancia, "White-coat hypertension, as defined by ambulatory blood pressure monitoring, and subclinical cardiac organ damage," Journal of Hypertension, vol. 33, no. 1, pp. 24-32, 2015.

[22] D. Tientcheu, C. Ayers, S. R. Das et al., "Target organ complications and cardiovascular events associated with masked hypertension and white-coat hypertension," Journal of the American College of Cardiology, vol. 66, no. 20, pp. 2159-2169, 2015.

[23] R. H. Fagard, H. Celis, L. Thijs et al., "Daytime and nighttime blood pressure as predictors of death and cause-specific cardiovascular events in hypertension," Hypertension, vol. 51, no. 1, pp. 55-61, 2008.

[24] T. W. Hansen, Y. Li, J. Boggia, L. Thijs, T. Richart, and J. A. Staessen, "Predictive role of the nighttime blood pressure," Hypertension, vol. 57, no. 1, pp. 3-10, 2011.

[25] A.-H. Investigators, G. C. Roush, R. H. Fagard et al., "Prognostic impact from clinic, daytime, and night-time systolic blood pressure in nine cohorts of 13,844 patients with hypertension," Journal of Hypertension, vol. 32, no. 12, pp. 2332-2340, 2014.

[26] J. J. Polónia, G. M. Gama, J. A. Silva, C. Amaral, L. R. Martins, and S. E. Bertoquini, "Sequential follow-up clinic and 
ambulatory blood pressure evaluation in a low risk population of white-coat hypertensive patients and in normotensives," Blood Pressure Monitoring, vol. 10, no. 2, pp. 57-64, 2005.

[27] Y. Huang, W. Huang, W. Mai et al., "White-coat hypertension is a risk factor for cardiovascular diseases and total mortality," Journal of Hypertension, vol. 35, no. 4, pp. 677-688, 2017.

[28] M. Abolbashari, "White coat hypertension and cardiovascular diseases: innocent or guilty," Current Cardiology Reports, vol. 20, no. 4, p. 25, 2018.

[29] G. Mancia and G. Grassi, "The heterogeneous nature of whitecoat hypertension," Journal of the American College of Cardiology, vol. 68, no. 19, pp. 2044-2046, 2016.

[30] T. G. Pickering, G. D. James, C. Boddie, G. A. Harshfield, S. Blank, and J. H. Laragh, "How common is white coat hypertension?" JAMA: The Journal of the American Medical Association, vol. 259, no. 2, pp. 225-228, 1988.

[31] R. H. Fagard, L. Thijs, J. A. Staessen, D. L. Clement, M. L. De Buyzere, and D. A. De Bacquer, "Prognostic significance of ambulatory blood pressure in hypertensive patients with history of cardiovascular disease," Blood Pressure Monitoring, vol. 13, no. 6, pp. 325-332, 2008.

[32] H.-Q. Fan, Y. Li, L. Thijs et al., "Prognostic value of isolated nocturnal hypertension on ambulatory measurement in 8711 individuals from 10 populations," Journal of Hypertension, vol. 28 , no. 10, pp. 2036-2045, 2010.

[33] S. Omboni, "A working definition of white-coat hypertension must include nocturnal blood pressure," The Journal of Clinical Hypertension, vol. 20, no. 8, p. 1183, 2018.

[34] D. Anstey, L. Colantonio, Y. Yano, J. Booth, and P. Muntner, "The importance of using 24-hour and nighttime blood pressure for the identification of white coat hypertension: data from the Jackson Heart Study," Journal of Hypertension, vol. 28, 2018.

[35] K. Asayama, L. Thijs, Y. Li et al., "Setting thresholds to varying blood pressure monitoring intervals differentially affects risk estimates associated with white-coat and masked hypertension in the population," Hypertension, vol. 64, no. 5, pp. 935-942, 2014.

[36] A. de la Sierra, E. Vinyoles, J. R. Banegas et al., "Prevalence and clinical characteristics of white-coat hypertension based on different definition criteria in untreated and treated patients," Journal of Hypertension, vol. 35, no. 12, pp. 2388-2394, 2017.

[37] G. Mancia, M. Bombelli, R. Facchetti et al., "Long-term risk of sustained hypertension in white-coat or masked hypertension," Hypertension, vol. 54, no. 2, pp. 226-232, 2009.

[38] O. Paez, P. Puleio, M. Gorocito, M. Visser, M. Schavione, and C. Majulmtsac, "Progression of white coat hypertension to sustained hypertension after 10 years," Argentine Journal of Cardiology, vol. 80, no. 3, 2012.

[39] G. Mancia, M. Bombelli, C. Cuspidi, R. Facchetti, and G. Grassi, "Cardiovascular risk associated with white-coat hypertension," Hypertension, vol. 70, no. 4, pp. 668-675, 2017.

[40] G. Mancia, R. Facchetti, G. Parati, and A. Zanchetti, "Effect of long-term antihypertensive treatment on white-coat hypertension," Hypertension, vol. 64, no. 6, pp. 1388-1398, 2014.

[41] G. Parati, L. Ulian, L. Sampieri et al., "Attenuation of the "white-coat effect" by antihypertensive treatment and regression of target organ damage," Hypertension, vol. 35, no. 2, pp. 614-620, 2000. 